\title{
Analyst
}

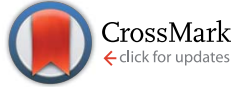

Cite this: Analyst, 2015, 140, 661

Received 5th October 2014

Accepted 17th November 2014

DOI: $10.1039 / c 4 a n 01794 c$

www.rsc.org/analyst

\section{Investigating acid-induced structural transitions of lysozyme in an electrospray ionization source $\uparrow$}

\begin{abstract}
Jong Wha Lee ${ }^{a}$ and Hugh I. Kim*ab
The effect of acids on the structure of lysozyme (Lyz) during electrospray ionization (ESI) was studied by comparing the solution and gas-phase structures of Lyz. Investigation using circular dichroism spectroscopy and small-angle X-ray scattering demonstrated that the folded conformation of Lyz was maintained in $\mathrm{pH} 2.2$ solutions containing different acids. On the other hand, analysis of the charge state distributions and ion mobility (IM) distributions, combined with molecular dynamics simulations, demonstrated that the gas phase structures of Lyz depend on the $p K_{a}$ of the acid used to acidify the protein solution. Formic acid and acetic acid, which are weak acids ( $\mathrm{p} K_{\mathrm{a}}>3.5$ ), induce unfolding of Lyz during ESI, presumably because the undissociated weak acids provide protons to maintain the acidic groups within Lyz protonated and prevent the formation of salt bridges. However, $\mathrm{HCl}$ suppressed the formation of the unfolded conformers because the acid is already dissociated in solution, and chloride anions within the ESI droplet can interact with Lyz to reduce the intramolecular electrostatic repulsion. These trends in the IM distributions are observed for all charge states, demonstrating the significance of the acid effect on the structure of Lyz during ESI.
\end{abstract}

\section{Introduction}

The structural transitions of proteins under non-native conditions have been actively investigated to understand protein stability ${ }^{1}$ and folding kinetics, ${ }^{2,3}$ as well as to identify folding intermediates. $^{3-5}$ The influence of organic solvents, high temperature, acidic $\mathrm{pH}$, molecular denaturants, and combinations of these denaturing conditions on protein structures has been examined. ${ }^{\mathbf{1 1 1}}$ In particular, protein denaturation under acidic conditions has gained attention because partially unfolded structures that are formed under acidic conditions are thought to have important implications in biological systems. $^{7-9,12}$

The structural dynamics of proteins have previously been analyzed via single molecule spectroscopy, ${ }^{13}$ sum frequency generation spectroscopy, ${ }^{14}$ as well as NMR spectroscopy. ${ }^{15}$ Mass spectrometry (MS) combined with electrospray ionization (ESI) has also been widely utilized for this purpose due to the soft properties of this technique. ${ }^{\mathbf{1 6 - 1 9}}$ Unsolvated proteins in the gas phase, formed via ESI, are known to retain their solution-like structures on a longer time-scale than is typically required for MS analysis, ${ }^{20}$ and these structures are thought to represent

${ }^{a}$ Department of Chemistry, Pohang University of Science and Technology (POSTECH), Pohang, 790-784, South Korea. E-mail: hughkim@postech.edu; Tel: +82-54-279-2341 ${ }^{b}$ Division of Advanced Materials Science, Pohang University of Science and Technology (POSTECH), Pohang, 790-784, South Korea

$\dagger$ Electronic supplementary information (ESI) available: Details on the experimental methods, additional discussions, and additional experimental data (Fig. S1-S8, Tables S1 and S2) are included. See DOI: 10.1039/c4an01794c different protein states that exist in solution. ${ }^{21}$ In addition, MS offers the unique capability to isolate and characterize particular structural components of proteins. ${ }^{21-25}$ For these reasons, the application of ESI-MS has been extended to the analysis of structural transitions of proteins. Charge state distributions (CSDs) in the ESI-MS spectra of a protein may serve as an indicator of its structural transition in solution. ${ }^{22,24-30}$ Protein denaturation is generally accompanied by an increase in the protein surface area and results in a shift of the CSD to higher charge states. ${ }^{26,29}$ Ion mobility (IM) measurements of gas-phase protein ions can also be used to deduce the structural transitions of proteins in solution. For example, a gradual change in the collision cross-section $\left(\Omega_{\mathrm{D}}\right)$ distribution of ubiquitin ions was observed as the amount of cosolvent was increased under acidic conditions. ${ }^{21}$

ESI of globular proteins is generally thought to follow the charged residue model (CRM), ${ }^{31,32}$ which proposes that solvent molecules in charged droplets undergo evaporation until Coulombic repulsion overcomes the surface tension of the droplets. The highly charged shrunken droplet then undergoes fission to generate smaller droplets. ${ }^{33}$ Evaporation and fission cycles continue until all of the solvent molecules evaporate and the excess charge remains on the protein. ${ }^{31}$ Because the proteins are subjected to non-native conditions during ESI, their structures may be influenced by a variety of factors ${ }^{27,34-40}$ and the proteins may adopt structures that are distinct from those in solution. ${ }^{\mathbf{4 1 , 4 2}}$ The native folding states may be maintained in the case of common native proteins with strong intramolecular interactions that can endure such non-native environments. ${ }^{20}$ 
However, the possible consequences of transferring non-native proteins with weak intramolecular interactions from solution to the gas phase are less well understood. ${ }^{37}$ It has previously been demonstrated that expansion of the protein structure due to Coulombic repulsion $^{37}$ and collapse of the extended structure may occur during or after ionization..$^{\mathbf{3 8 3 - 4 5}}$ Thus, to obtain reliable data on protein structures in solution, it is important to investigate the factors that influence the protein structure during transfer to the gas phase.

Hen egg white lysozyme (Lyz; molecular weight: $14.3 \mathrm{kDa}$ ) is a small protein with 129 amino acid residues that functions primarily to degrade bacterial cell walls to prevent bacterial infection. ${ }^{46}$ Lyz has been widely characterized both in solution and in the gas phase, ${ }^{1-6,43,47-54}$ and is known to be very stable to acid-induced denaturation, maintaining its folded state at $\mathrm{pH}$ levels as low as 1.2..$^{5}$ Therefore, Lyz is an ideal model for evaluation of the effects of acids on the protein structure during ESI, independently from the conformational effects in solution.

In the present study, the structural transitions of Lyz during ESI, induced by different acids ( $\mathrm{HCl}$; formic acid, FA; and acetic acid, AA), are investigated. The solution-phase structures of Lyz are probed using circular dichroism (CD) spectroscopy and synchrotron small-angle X-ray scattering (SAXS), combined with computational sampling of Lyz structures using molecular dynamics (MD) simulations. ESI-IM-MS, combined with MD simulations in the gas phase, is then utilized to investigate the structures of Lyz in the gas phase. The observed phenomena are explained in relation to the ion-pairing phenomenon during ESI and the difference in the $\mathrm{p} K_{\mathrm{a}}$.

\section{Experimental}

\subsection{Sample preparation}

Lyz (from chicken egg white) and all other materials were purchased from Sigma-Aldrich (Saint Louis, MO, USA) unless stated otherwise. HPLC-grade water was purchased from Avantor Performance Materials, Inc. (Center Valley, PA, USA) and used as a solvent. The $\mathrm{pH}$ of the solutions was measured with an Orion 3 Star $\mathrm{pH}$ meter (Thermo Scientific, San Jose, CA, USA). The concentrations of FA and AA were approximately $1 \% \mathrm{v} / \mathrm{v}$ and $10 \% \mathrm{v} / \mathrm{v}$, respectively, for $\mathrm{pH} 2.2$ solutions.

\subsection{CD spectroscopy}

CD spectroscopy was performed using a Jasco J-815 polarimeter (Easton, MD, USA). CD signals were measured at a speed of $50 \mathrm{~nm} \mathrm{~min} \mathrm{~min}^{-1}$ and averaged for fifteen repetitions. The concentration of the Lyz samples was $10 \mu \mathrm{M}$.

\subsection{SAXS}

All SAXS measurements were carried out at the 4C SAXS II beamline of the Pohang Accelerator Laboratory (PAL) using a Lyz concentration of $2 \mathrm{mg} \mathrm{mL}^{-1}$ and a sample-to-detector distance of $2 \mathrm{~m}$. The scattering intensity, $I(q)$, was obtained with scattering vectors $(q=(4 \pi / \lambda) \sin \theta)$ ranging from 0.01 to
$0.345 \AA^{-1}$. All scattering experiments were performed at $20{ }^{\circ} \mathrm{C}$. At least three fresh samples were prepared, and each scattering pattern was recorded over $20 \mathrm{~s}$. The data were analyzed using ATSAS 2.5.2. ${ }^{55-58}$ The Guinier relationship (eqn (1)) was used to obtain the radius of gyration $\left(R_{\mathrm{g}}\right)$ values for Lyz. ${ }^{59}$

$$
\ln [I(q)]=\ln [I(0)]-R_{\mathrm{g}}{ }^{2} q^{2} / 3
$$

\subsection{ESI-MS}

ESI-IM-MS experiments were performed with a Waters Synapt G2 HDMS quadrupole time-of-flight (Q-TOF) mass spectrometer with travelling wave ion mobility spectrometry (TWIMS) capability. A $Z$-spray ESI source was used with capillary, sample cone, and extraction cone voltages of $2.00 \mathrm{kV}, 40 \mathrm{~V}$, and $2.00 \mathrm{~V}$, respectively. The source temperature was $50{ }^{\circ} \mathrm{C}$, the desolvation temperature was $50{ }^{\circ} \mathrm{C}$, and the desolvation gas flow rate was $200 \mathrm{~L} \mathrm{~min}^{-1}$. The gas flows for the helium and drift cells were $100 \mathrm{~mL} \mathrm{~min}{ }^{-1}$ and $80 \mathrm{~mL} \mathrm{~min}^{-1}$, respectively, which provide a pressure of 2.49 mbar for the drift cell. The wave velocity and height were $270 \mathrm{~m} \mathrm{~s}^{-1}$ and $13 \mathrm{~V}$, respectively. All conditions were tested from gentle to harsh to ensure that gentle conditions are used. The arrival times from the IM experiments were converted into helium $\Omega_{\mathrm{D}}$ values by calibration using denatured ubiquitin, cytochrome c, and apomyoglobin. ${ }^{60,61}$

Q-Orbitrap experiments were performed with a Thermo Scientific Q-Exactive (San Jose, CA, USA) instrument. The spray voltage, capillary temperature, and sheath gas flow were $4 \mathrm{kV}, 200{ }^{\circ} \mathrm{C}$, and 10 (arbitrary units), respectively. The instrument was calibrated immediately before the experiments.

\subsection{Computational modeling}

MD simulations of the Lyz structures were performed with Gromacs 4.5.5 (ref. 62) and the all-atom OPLS force field, ${ }^{63}$ starting from the crystal structure of Lyz. $^{48}$ GNOM $^{56}$ and GASBOR $^{57}$ were used to obtain $a b$ initio structures of Lyz in solution based on the SAXS curves. For molecular modeling of Lyz from the SAXS curves, a very large number (>200 000) of Lyz conformations was generated with simulated annealing. Theoretical scattering curves of the candidate structures were generated with CRYSOL $^{58}$ and compared with the experimental scattering curves.

Prior to all gas-phase simulations, Lyz was first energyminimized with steepest descent minimization. For constant temperature simulations, Lyz was heated from 0 to $300 \mathrm{~K}$ in $100 \mathrm{ps}$ and simulated at $300 \mathrm{~K}$ for $29.9 \mathrm{~ns}$. For simulated annealing, Lyz was subjected to 100 annealing cycles between 300 and $1000 \mathrm{~K}$. The charge site configuration of Lyz was assigned to minimize the sum of the Coulombic repulsion between charged residues. Theoretical $\Omega_{\mathrm{D}}$ values were calculated using the exact hard-sphere scattering (EHSS) model ${ }^{64}$ and compared with the values from the trajectory (TJ) model. ${ }^{65}$ Further details of the computational procedures are presented in the ESI. $\dagger$ 


\section{Results and discussion}

\subsection{Structure of Lyz in solution}

Lyz is known to be highly resistant to acid-induced denaturation in solution. ${ }^{5} \mathrm{CD}$ analysis of $\mathrm{Lyz}$ in $\mathrm{HCl}$ and FA solution at $\mathrm{pH} 2.2$ demonstrated that the native secondary structures were maintained (Fig. 1a). Although the strong absorption of AA in the far$\mathrm{UV}$ region prohibits $\mathrm{CD}$ measurements in AA solution, the CD experiments in $\mathrm{HCl}$ and $\mathrm{FA}$ solution indicate that the structure of Lyz is highly stable in acidic solutions.

SAXS experiments can provide more detailed information on the overall size, shape, and folding state of proteins. The SAXS curves $(\ln [I(q)]$ versus $q)$ of Lyz in different solutions are shown in Fig. $1 \mathrm{~b}$. The $R_{\mathrm{g}}$ of a protein in solution can be derived from a Guinier plot $\left(\ln [I(q)]\right.$ versus $\left.q^{2}\right)$ of the SAXS curve when fitted to a linear curve (eqn (1)). ${ }^{59}$ The Guinier plots for all samples showed good linearity in the small- $q$ range, verifying the homogeneity of the Lyz solutions (Fig. 1c). The $R_{\mathrm{g}}$ values for Lyz in pure water (HPLC-grade, pH 4.8), $20 \mathrm{mM}$ sodium phosphate buffer solution ( $\mathrm{pH}$ 7), and $50 \mathrm{mM}$ ammonium acetate buffer solution ( $\mathrm{pH}$ 7) are all $\sim 15.3 \AA$, in good agreement with those previously reported for the native Lyz monomer (Table 1)..$^{\mathbf{4 , 6 4 9 , 5 8}}$ No significant change in the $R_{\mathrm{g}}$ value of Lyz was observed in acidic solutions ( $\mathrm{pH}$ 2.2), regardless of the acid used to adjust the $\mathrm{pH}$, which demonstrates that the tertiary structure of Lyz is maintained in solution at pH 2.2. Moreover, bell-shaped Kratky plots $\left(q^{2}[I(q)]\right.$ versus $\left.q\right)$ were obtained for Lyz in all solutions, indicating that the protein assumes a globular, compact structure (Fig. 1d). ${ }^{59}$ The $R_{\mathrm{g}}$ values from the Guinier plots and the data from the Kratky plots are summarized in Table 1.
Table $1 \quad R_{\mathrm{g}}$ and $R^{2}$ values ${ }^{a}$ and folding states ${ }^{b}$ of Lyz

\begin{tabular}{|c|c|c|c|}
\hline Solution $(\mathrm{pH})$ & $R_{g} / \AA$ & $R^{2}$ & Folding state \\
\hline Water (4.8) & 15.4 & 0.97 & Globular \\
\hline Water/SPB ${ }^{c}(7)$ & 15.3 & 0.97 & Globular \\
\hline Water/AmAc ${ }^{d}(7)$ & 15.3 & 0.97 & Globular \\
\hline Water/HCl (2.2) & 15.4 & 0.96 & Globular \\
\hline Water/FA (2.2) & 15.3 & 0.94 & Globular \\
\hline Water/AA (2.2) & 15.5 & 0.96 & Globular \\
\hline
\end{tabular}

${ }^{a}$ Obtained from linear fitting curves of Guinier plots (Fig. 1c). ${ }^{b}$ Obtained from Kratky plots (Fig. 1d). ${ }^{c}$ SPB: $20 \mathrm{mM}$ sodium phosphate buffer with $100 \mathrm{mM}$ NaCl. ${ }^{d}$ AmAc: $50 \mathrm{mM}$ ammonium acetate buffer.

Fig. 1e shows the $a b$ initio envelopes of Lyz in solution, constructed from the SAXS curves. ${ }^{57}$ The $a b$ initio envelopes of Lyz confirm that the compact, globular shape of Lyz is maintained in water at pH 2.2 regardless of the acid used to adjust the $\mathrm{pH}$. To investigate its conformation at the molecular level, molecular structures of Lyz in water at pHs 7 and 2.2 were sampled by simulated annealing, and the theoretical scattering curves were then compared with the experimental SAXS curves. ${ }^{58}$ Fig. 1f shows the candidate structures that have the minimum discrepancy $(\chi)$ relative to the experimental SAXS curves. All of the computationally modeled structures were essentially compact and globular. Moreover, the secondary structural features of the structures were similar to that of Lyz in its native folding state. Only minor differences in the assignments of the turns were observed at the C-termini (Fig. S3 in the $\mathrm{ESI} \dagger)$. a)

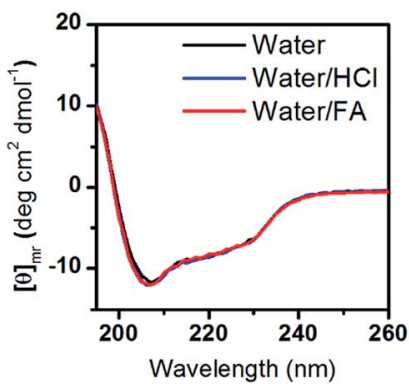

d)

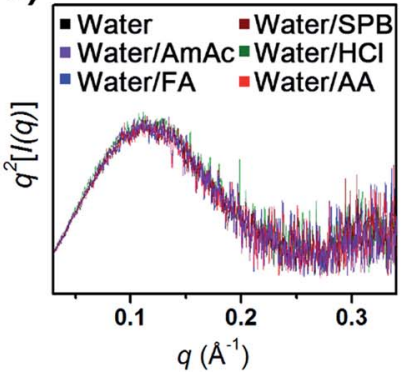

b)

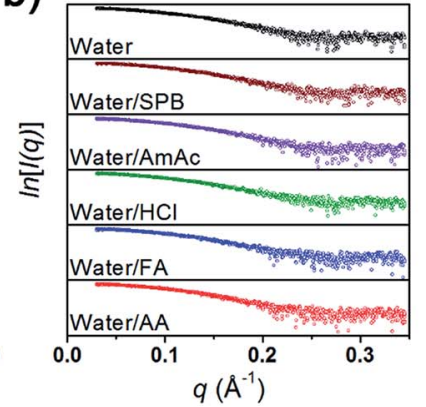

e)

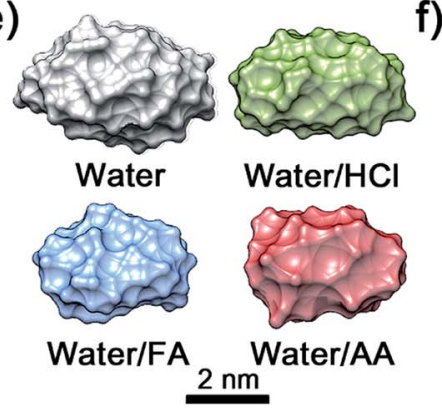

c)

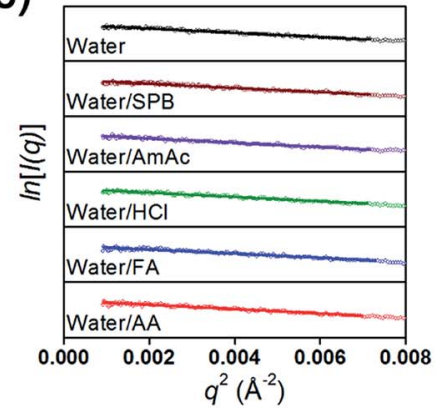

f)

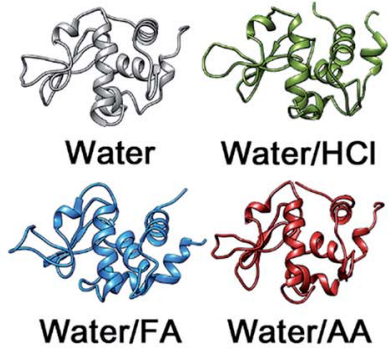

Fig. 1 (a) CD spectra of Lyz, (b) SAXS curves, (c) Guinier plots and linear fitting of SAXS curves, (d) Kratky plots of SAXS curves, (e) solution structures of Lyz reconstructed from SAXS curves using GASBOR, and (f) best-fit molecular models of Lyz using CRYSOL. The pH of the acidic solutions was 2.2. See Fig. S1 $\uparrow$ for fitting of the theoretical scattering curves to the experimental scattering curves. Additional candidate structures are presented in Fig. S2. $\dagger$ 


\subsection{Structure of Lyz in the gas phase}

3.2.1. CSDs of Lyz. Herein, for discussion of the MS experiments, focus is placed on the CSD and $\Omega_{\mathrm{D}}$ distributions of the Lyz ions obtained using pure water ( $\mathrm{pH} 5$ ) and not from the buffer solution to rule out the effects of solution additives on the CSD. ${ }^{66}$ Fig. 2a shows that the Lyz ions generated from pure water have a unimodal CSD centered at the +10 charge state, which is similar to the previously reported spectra of Lyz in water. $^{27,43,66}$ This charge state is consistent with that predicted by the Rayleigh-limit charge theory $(+9.3),{ }^{66}$ and also with the prediction from the conformation-dependent charge neutralization theory, ${ }^{67}$ where a charge state of +9 is predicted for Lyz because it possesses nineteen basic residues (six lysines, eleven arginines, one histidine, and the $\mathrm{N}$-terminus) and ten acidic residues (seven aspartic acids, two glutamic acids, and the $\mathrm{C}$ terminus). Addition of acid to the solution shifted the CSD. A bimodal CSD was obtained for Lyz from aqueous $\mathrm{HCl}$, with peaks centered at the +8 and +11 charge states, whereas the CSDs of Lyz from FA and AA solutions were centered at the +9 charge state. It is well known that if denaturation does not occur, addition of acids generally shifts the CSDs to lower charge states. ${ }^{68}$ This explains the shift of the CSD for Lyz from FA and AA solutions. However, the bimodal CSD of Lyz from $\mathrm{HCl}$ solution does not conform to this trend. Bimodal CSDs are generally attributed to the presence of distinct conformers in solution; ${ }^{26}$ however, the present SAXS analysis indicates that the native folding of Lyz is maintained in $\mathrm{HCl}$ solution at $\mathrm{pH}$ 2.2. Protein denaturation during ESI may give rise to multimodal CSDs, but such denaturation generally leads to new distributions at higher charge states in the MS spectrum. ${ }^{27,34-36,39}$ In contrast, the new distribution for Lyz from $\mathrm{HCl}$ solution was centered at a lower charge state.

The veracity of the ESI-MS spectra acquired using the Q-TOF mass spectrometer (Waters Synapt G2 HDMS) was evaluated by comparison with the ESI-MS spectra acquired using a Q-Orbitrap mass spectrometer (Thermo Q-Exactive). The ESI-MS spectra of Lyz from pure water, FA solution, and AA solution obtained with the Q-Orbitrap show high similarity with those obtained with the Q-TOF. A CSD centered at the +10 charge state was observed for Lyz from pure water, and there was a shift to lower charge states with addition of FA or AA (Fig. 2b). However, the bimodal CSD was not observed in the spectrum for Lyz from $\mathrm{HCl}$ solution. Instead, numerous peaks corresponding to Lyz adducted with chloride were observed for the +7 and +8 charge states, whereas adduct formation was largely undetected in the spectrum acquired with the Q-TOF (Fig. 2a). As the chloride adduct peaks appear to be affecting the CSD, the intensity of all the peaks of Lyz from $\mathrm{HCl}$ solution for each charge state was integrated as shown in Fig. 2c. The result presented in Fig. 2c shows that the total intensity of the +8 charged Lyz is greater than that of the +11 charged ion. Moreover, the overall profile presented in Fig. $2 \mathrm{c}$ is similar to the spectrum of Lyz from $\mathrm{HCl}$ solution acquired using the Q-TOF (Fig. 2a). This observation indicates that the bimodal CSD obtained using the Q-TOF was due to the efficient removal of adduct peaks during ion transmission, which yielded unadducted peaks with high intensity. Overall, the CSD of Lyz in aqueous solution is governed by the presence of solution additives, which is consistent with previous reports on the CSD of Lyz. ${ }^{66}$

3.2.2. IM distributions of Lyz. The IM distributions of the Lyz ions were then examined using TWIMS to investigate the correlation between the structure of Lyz in solution and the gas phase. It is not possible to assign a charge state that represents a solution-phase conformation of Lyz because it was observed that the charge state of Lyz depends on the competition for protons in the late stages of $\mathrm{ESI}^{66}$ rather than on the structure in solution. Therefore, we discuss the IM distribution of all observable charge states. It was observed that the IM
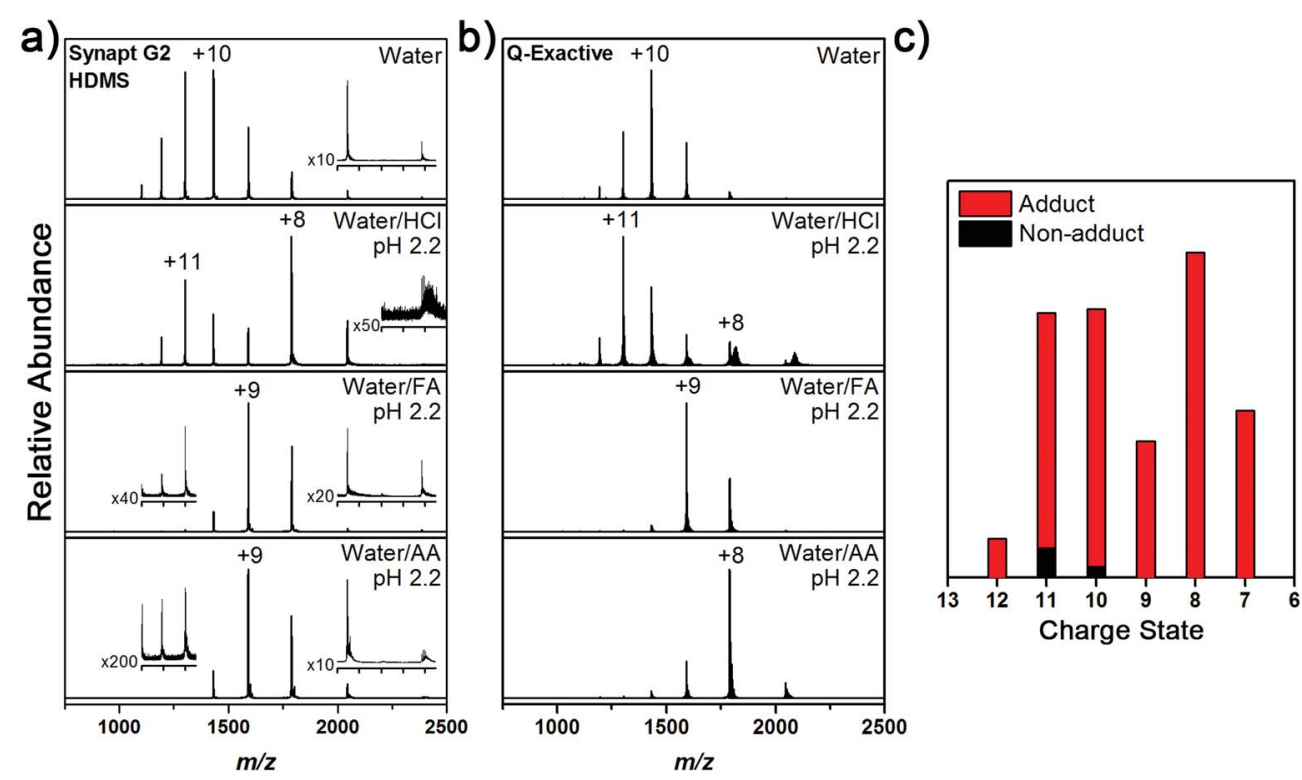

Fig. 2 ESI-MS spectra of Lyz acquired with the: (a) Synapt G2 HDMS and (b) Q-Exactive. (c) Integrated signal intensities for each charge state in the spectrum of $\mathrm{Lyz}$ in $\mathrm{HCl}$ solution acquired using the $\mathrm{Q}$-Exactive. 
distributions for all charge states, regardless of their abundance, followed identical trends. Fig. 3 shows the IM distributions for the +6 to +12 charged Lyz ions, plotted as $\Omega_{\mathrm{D}}$ values. The $\Omega_{\mathrm{D}}$ distribution of the +6 charge state of Lyz from water was unimodal with a $\Omega_{\mathrm{D}}$ of $1342 \AA^{2}$. The $\Omega_{\mathrm{D}}$ distribution for the +7 charged Lyz ions was bimodal, displaying a distribution with a small $\Omega_{\mathrm{D}}\left(1382 \AA^{2}\right)$ and a less abundant distribution with a greater $\Omega_{\mathrm{D}}\left(1666 \AA^{2}\right)$. The $\Omega_{\mathrm{D}}$ values at the +6 and +7 charge states are comparable to those of the compact conformers reported in a prior drift-tube IMS (DTIMS) study. ${ }^{50}$ The $\Omega_{\mathrm{D}}$ of Lyz at +8 or higher charge states was significantly greater $\left(>1750 \AA^{2}\right)$ than that of Lyz at lower charge states and is closer to the $\Omega_{\mathrm{D}}$ of Lyz ions that have undergone gas-phase unfolding with high energy injections (see Table S1 in the ESI $\dagger$ for comparison). ${ }^{50}$ This suggests that gas-phase unfolding of +8 and more highly charged Lyz ions is triggered during the IM-MS experiments. Although it is difficult to specify the stage during IM-MS at which unfolding occurs, it is unlikely that unfolding occurs during ESI, since the addition of ammonium acetate, which favors the formation of native-like ions, ${ }^{69,70}$ does not have any effect on the IM distributions (Fig. S4 in the ESI $\dagger$ ). As other possibilities, unfolding may be due to RF heating of Lyz ions ${ }^{54}$ or heating during injection into the TWIMS cell. ${ }^{71}$ A number of studies employing different instruments ${ }^{\mathbf{4 3 5 , 7 2}}$ have also reported large change in the $\Omega_{\mathrm{D}}$ values between +7 and +8 charged Lyz ions. This change indicates that compact Lyz conformers with +8 or higher charge states, which were observed using DTIMS, are meta-stable in the gas phase and, thus, are sensitive to heating in the gas phase.

Nevertheless, the IM distributions of the Lyz ions from $\mathrm{HCl}$ solution were similar to those from water, in line with the native structure of Lyz in solution (Fig. 3). In contrast, significantly different distributions were observed for the Lyz ions from the FA and AA solutions. The IM distribution for the +6 charged Lyz ions from the FA solution exhibited an extended conformation $\left(1482 \AA^{2}\right)$ in addition to a compact conformation $\left(1332 \AA^{2}\right)$. An increase in the abundance of the extended conformer $\left(1654 \AA^{2}\right)$ was also observed for the +7 charged Lyz ion from the FA solution. Lyz ions at the +6 charge state from the AA solution also exhibited extended conformations with a $\Omega_{\mathrm{D}}$ of $1488 \AA^{2}$. Extended conformers were also observed for highly charged $(+10$ to +12$)$ ions from the FA and AA solutions. Because the compact structure of Lyz is unperturbed in the aqueous acid solutions, the additional conformers observed from the FA and AA solutions indicate that the formation of the extended conformers is facilitated by the presence of FA and AA during ESI. Although it was noted that gas-phase unfolding of Lyz can occur for the +8 and higher charge states, the acid effect leading to the formation of the unfolded conformers must be operative during ESI, since acids cannot affect the structure of Lyz after complete desolvation.

The plot of $\Omega_{\mathrm{D}}$ versus the charge state presented in Fig. $4 \mathrm{a}$ suggests that there are distinct conformational states of Lyz ions. Firstly, there are ions with a compact conformation at the +6 and +7 charge states (A class). Upon unfolding, they adopt a more extended conformation (B class). B class ions exist for all the charge states, and $\Omega_{\mathrm{D}}$ increases gradually as the charge of the Lyz ions increases. As discussed earlier, structural rearrangement of the Lyz ions occurs during IM-MS, and thus the Lyz ions from pure water with +8 or higher charge states are exclusively B class ions. The spontaneous structural rearrangement of these relatively highly charged ions suggests that B class structures are more energetically favored in the gas phase than the A class structures, which are more compact and solution-like. However, the low charge state $(+6$ and +7$)$ ions do not readily undergo transition into $\mathrm{B}$ class ions, which indicates that charge-charge repulsion is important for surmounting the energy barrier between A class and B class structures. The abundance of B class ions increases with the use of FA and AA (especially FA) for +6 and +7 charged ions, which implies that FA

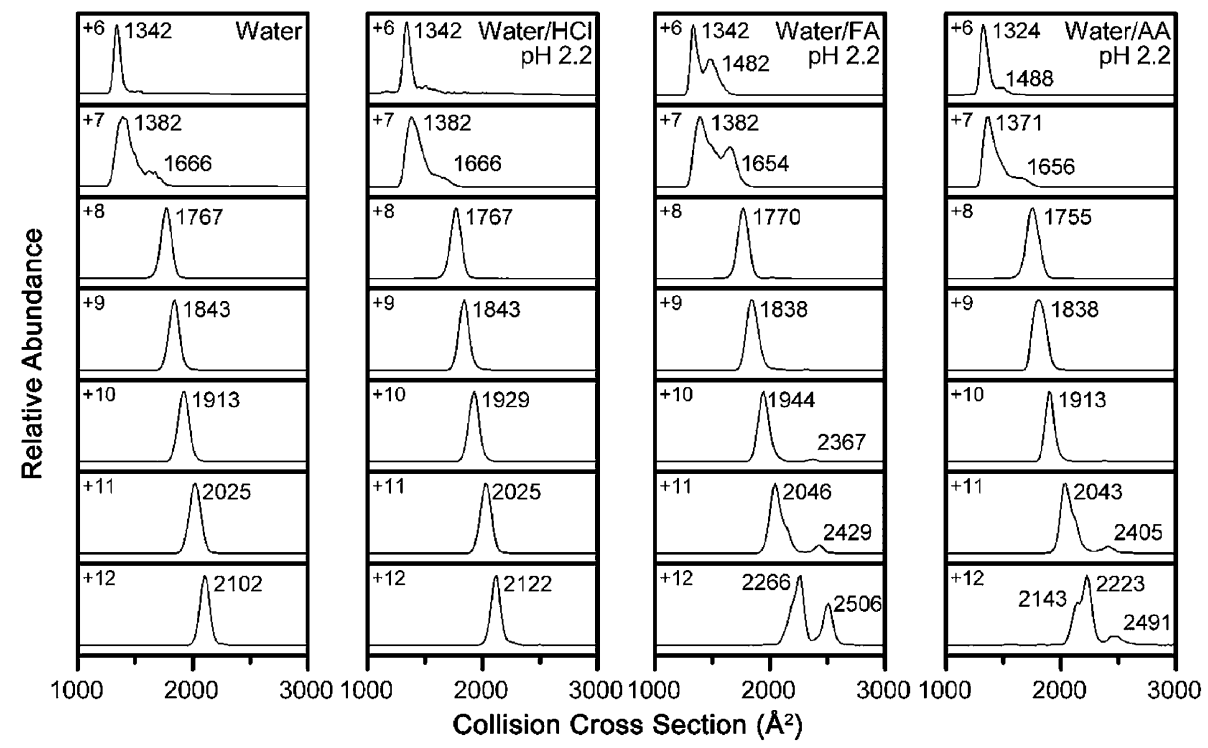

Fig. 3 IM spectra of +6 to +12 charged Lyz ions. The $\mathrm{pH}$ of the acidic solutions was 2.2 . 
a)

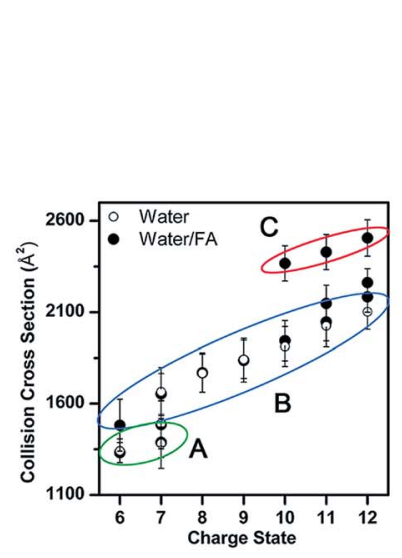

b)

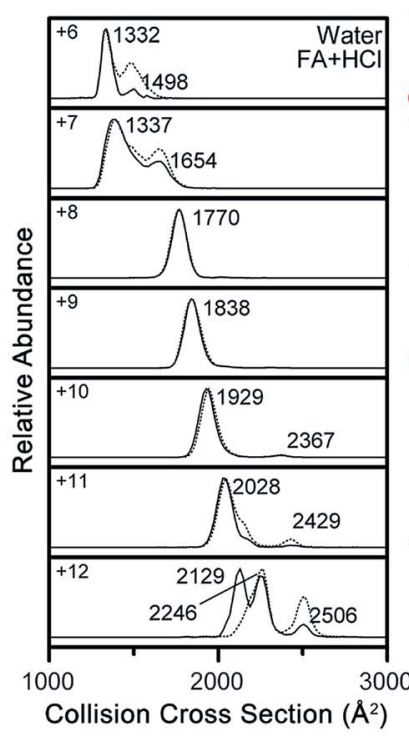

c)

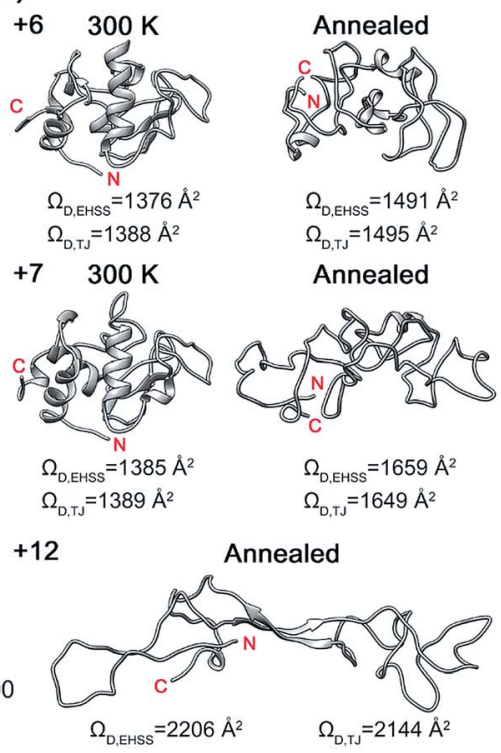

Fig. 4 (a) Plot of $\Omega_{\mathrm{D}}$ versus charge state for Lyz ions from water and water/FA solution. The error bars indicate the full-width-half-maximum of each peak in the IM distributions. (b) IM spectra of +6 to +12 charge states of Lyz from aqueous solution with $0.1 \% \mathrm{v} / \mathrm{V} F A$ (dots) and $0.1 \%$ v/v FA with $\mathrm{HCl}$ at pH 2.2 (solid lines). See Fig. S5 in the ESI $\uparrow$ for the MS spectra. (c) Structures of +6 and +7 charged Lyz ions from simulation at $300 \mathrm{~K}$, and the representative structures of $+6,+7$, and +12 charged Lyz ions from simulated annealing, with $\Omega_{\mathrm{D}}$ values from EHSS and TJ models

and AA can lower the energy barrier and facilitate transition into B class structures.

The acid effect becomes more significant for the more highly charged $(+10,+11$, and +12$)$ Lyz ions. As illustrated in Fig. $4 \mathrm{a}$, the highly charged Lyz ions from the FA and AA solutions adopt a more unfolded structure, categorized as $\mathrm{C}$ class, which was not observed in other solutions. The absence of $\mathrm{C}$ class structures for Lyz from pure water suggests that the energy barrier for the transition into these structures is very high. It appears that a combination of electrostatic repulsion (high charge state) and the FA and AA effect facilitates the transition into $\mathrm{C}$ class ions.

\subsection{Effects of other acids during transfer to the gas phase}

To understand the effect of acids on the structure of Lyz ions during ESI, additional ESI-IM-MS experiments were performed with acids of various $\mathrm{p} K_{\mathrm{a}} \mathrm{s}$. Table 2 summarizes the results from ESI-IM-MS experiments of Lyz at $\mathrm{pH} \sim 2.2$, where the $\mathrm{pH}$ was adjusted with various acids. Weak acids with a high $\mathrm{p} K_{\mathrm{a}} \quad(>3.5)$ facilitated the formation of unfolded conformers. The high $\mathrm{p} K_{\mathrm{a}}$ requirements may explain the absence of unfolded conformers with the use of $\mathrm{HCl}$ solutions. To test the effect of $\mathrm{HCl}$ on the structure of the Lyz ion during ionization, Lyz in an aqueous solution containing both FA and $\mathrm{HCl}$ was electrosprayed. $\mathrm{HCl}$ was added to the Lyz solution containing $0.1 \% \mathrm{v} / \mathrm{v} \mathrm{FA}(\mathrm{pH} 2.7)$ to reach a $\mathrm{pH}$ of 2.2 , and the ESI-IM-MS results were compared with that using only $0.1 \% \mathrm{v} / \mathrm{v}$ FA. Fig. $4 \mathrm{~b}$ shows that the abundance of the unfolded conformers was reduced by addition of $\mathrm{HCl}$. Thus, it was found that $\mathrm{HCl}$ actually suppresses the formation of extended conformers.

\subsection{Theoretical structure of Lyz ions in the gas phase}

MD simulations were performed to gain insight into the conformation of Lyz ions in the gas phase. The $\Omega_{\mathrm{D}}$ for the Lyz crystal structure is $1563 \AA^{2}$; this value was reduced to $\sim 1380 \AA^{2}$ for +6 and +7 charged Lyz after a $30 \mathrm{~ns}$ simulation at $300 \mathrm{~K}$ (Fig. S6 in the ESI†). This value is in good agreement with the experimental $\Omega_{\mathrm{D}}$ of the A class ions (Fig. 4a). The simulated structures (Fig. 4c) show that the compact Lyz conformer at +6 and +7 charge states can maintain its native-like structure (i.e. A class) in the gas phase. Because no significant unfolding of Lyz occurred in the simulation at $300 \mathrm{~K}$ even for the highly charged ions, simulated annealing was performed to gain insight into the conformation of unfolded Lyz ions in the gas phase. The scatter plot of energy versus $\Omega_{\mathrm{D}}$ for each sampled charge state (Fig. S7 in the ESI $\dagger$ ) produced a single domain with $\Omega_{\mathrm{D}}$ values similar to the experimental values for the B class ions (Table S2 in the ESI $\dagger$ ), which indicates that Lyz is present as the partially unfolded conformation (i.e., B class) even after thermal denaturation. It can be inferred that the four intramolecular disulfide bonds of Lyz prevent complete denaturation. The $+6,+7$, and +12 charged Lyz with theoretical $\Omega_{\mathrm{D}}$ values similar to the experimental values and low energy are shown in Fig. 4c. The structures for other charge states are presented in Fig. S8 in the ESI. $\uparrow$ The $\Omega_{\mathrm{D}}$ values obtained using the EHSS and TJ models agree within $3 \%$, indicating that the theoretical $\Omega_{\mathrm{D}}$ values are reliable. It is observed that structures from the simulated annealing are more loosely structured with smaller secondary structural contents than the A class ions at +6 and +7 charge states. Higher charge state ions adopt more elongated structures than the congeners in lower charge states. However, even these ions are not fully elongated, which can be seen from the 
Table 2 Acids used for observation of the extended structure via IM spectra of Lyz at pH 2.2 in water

\begin{tabular}{llll}
\hline Acids & $\mathrm{p} K_{\mathrm{a}}$ & Observed charge states & Extended conformers $^{a}$ \\
\hline Hydrochloric acid & -10 & $+6 \sim+12$ & N.O. \\
Trifluoroacetic acid & 0.23 & $+6 \sim+12$ & N.O. \\
Dichloroacetic acid & 1.25 & $+6 \sim+12$ & N.O. \\
Chloroacetic acid & 2.87 & $+7 \sim+13$ & N.O. \\
L-Tartaric acid & $2.89 / 4.40$ & $+9 \sim+14$ & N.O. \\
Fumaric acid (pH 2.3$)^{b}$ & $3.03 / 4.44$ & $+8 \sim+14$ & N.O. \\
Citric acid & $3.14 / 4.75 / 6.39$ & $+10 \sim+12$ & N.O. \\
Formic acid & 3.77 & $+6 \sim+12$ & $+6,+7,+10,+11,+12$ \\
LD-Lactic acid & 3.86 & $+10 \sim+13$ & $+12,+13$ \\
Acetic acid & 4.76 & $+6 \sim+13$ & $+6,+11,+12,+13$ \\
${ }^{a}$ N.O. $=$ not observed. ${ }^{b}$ Due to the limited solubility, the experiment was performed at pH 2.3. &
\end{tabular}

agreement between their $\Omega_{\mathrm{D}}$ values with the experimental values for the B class ions (Table $\mathrm{S} 2$ in the $\mathrm{ESI} \dagger$ ). The results from MD simulations imply that the transition from the most compact conformations (i.e., A class) to partially unfolded states (i.e., B class) is possible by structural rearrangement in the gas phase. ${ }^{50,54}$ Further unfolding with more significant change in the intramolecular interactions appears to be necessary to yield C class ions with a very high $\Omega_{\mathrm{D}}\left(2506 \AA^{2}\right.$, Fig. 3$)$. However, these states were inaccessible even with annealing cycles at a very high temperature $(1000 \mathrm{~K})$.

\subsection{Structural transition during transfer to the gas phase}

In the positively charged droplets generated by ESI, cationic species, including protons, are generally considered to be localized on the droplet surface to minimize electrostatic repulsion, ${ }^{32}$ while the anions would be present on a relatively inner side of the droplet. Thus, when Lyz solution is acidified with $\mathrm{HCl}$, the chloride anions would be proximate to Lyz as the protein exists within the ESI droplet. ${ }^{32}$ The concentration of chloride would further increase with shrinkage of the droplet. Then, the chloride anions can form ion pairs with the positively charged sites of Lyz during ESI. Such charge solvation by anions is well known to be capable of inducing protein folding. For example, cytochrome $\mathrm{c}$ is maximally denatured in solution at pH 2, but is folded into a molten globule state with further addition of $\mathrm{HCl}^{.9,73}$ This is because most of the sites available in the protein are already protonated at $\mathrm{pH} 2$, and further lowering the $\mathrm{pH}$ increases the concentration of chloride ions available to interact with the protein and reduces the electrostatic repulsion between the charged sites of the protein. ${ }^{9,73}$ Moreover, charge solvation can induce protein folding in the gas phase $e^{72,74}$ by reducing the intramolecular electrostatic repulsion. The existence of a significant interaction between Lyz and chloride ions during ESI is demonstrated herein by the extensive chloride adduction observed in Fig. $2 \mathrm{~b}$ and is also supported by a previous study which showed that Lyz in solution at $\mathrm{pH} 2.5$ can interact with up to 12 chloride ions. ${ }^{75}$ The reduction in the electrostatic repulsion due to interactions with chloride explains why addition of $\mathrm{HCl}$ to the FA solution suppressed the formation of unfolded Lyz conformers (Fig. 4b).
In contrast, when weak acids are used for acidification, their undissociated forms would be dispersed throughout the droplet in excess. The undissociated acids may donate their protons to neutralize the acidic groups of Lyz. The salt bridges in Lyz may be neutralized due to this process. Then, as the solvent evaporates, the increase in the electrostatic interaction due to the loss of the dielectric environment, or heating of the droplet, may cause Lyz to adopt a conformation that is different from that adopted by Lyz from the pure water droplet. This is in accordance with previous studies on ion-pairing during ESI. ${ }^{76-79}$ It is well-documented that the anion of trifluoroacetic acid $\left(\mathrm{p} K_{\mathrm{a}}=0.23\right)$ forms ion pairs with analytes and suppresses overall signals during liquid chromatography (LC)-MS. ${ }^{76-79}$ This can be prevented by the addition of weak acids, which maintains the acidity of the droplet. ${ }^{76,77}$ The effectiveness of acid-induced refolding would be much smaller for formate and acetate than chloride, because the effectiveness follows the electroselectivity of the anion-exchange resins toward anions. ${ }^{73,80}$ On this basis, acetate would be much less effective than chloride for solvating the positive charge on Lyz. ${ }^{80}$ Although the data for formate are unavailable, its properties can be expected to be more similar to those of acetate than chloride.

It is possible that the composition of FA and AA in the ESI droplet changes as evaporation occurs. For $\mathrm{HCl}$, the concentration of chloride anions is expected to increase as the droplet evaporates. However, in the case of the droplets containing FA and AA, the composition is more complex to predict because the acids are volatile. The respective vapor pressures of water, ${ }^{81} \mathrm{FA},{ }^{82}$ and $\mathrm{AA}^{83}$ are 31,72 , and 27 mbar at $300 \mathrm{~K}$. An elegant study by Grimm and Beauchamp demonstrated that the more volatile component in a cosolvent system evaporates preferentially from a charged droplet. ${ }^{33} \mathrm{On}$ the basis of their study, FA would be preferentially evaporated and depleted from the ESI droplet, while the fraction of AA would increase or at least remain constant. However, it was observed herein that the CSDs and IM distributions are affected by addition of acids. These facts indicate that the influence of acids on macromolecules during ESI may be persistent. 


\section{Conclusions}

The structural transitions of Lyz during transfer from solution to the gas phase via ESI were investigated. The IM spectra of Lyz varied depending on the acid utilized in the solution, in contrast to the structure of Lyz in solution. A consistent trend in the $\Omega_{\mathrm{D}}$ distributions was observed for all charge states, where FA and AA promoted the formation of unfolded Lyz ions, while $\mathrm{HCl}$ suppressed the formation of unfolded Lyz ions. It is proposed that ion-pairing by the anions of strong acids promotes the formation of compact Lyz conformers. On the other hand, charging of Lyz by an excess of undissociated weak acids is thought to influence the structure of Lyz during ESI. To the best of our knowledge, this is one of the first comprehensive studies on the effect of acids during ESI. We believe that further investigations on the factors that influence protein structures during ESI will greatly aid in the development of a reliable method to probe the solution-phase structural distribution of proteins using ESI-IM-MS. The effect of organic solvents on protein structures during ESI is currently under investigation.

\section{Acknowledgements}

This work was supported by a Basic Research Program (grant no. 2013R1A1A2008974) through the National Research Foundation (NRF) of Korea funded by the Ministry of Science, ICT, and Future Planning (MSIP) and a grant of the Korea Health Technology R\&D Project through the Korea Health Industry Development Institute (KHIDI), funded by the Ministry of Health \& Welfare of Korea (grant no. HT13C-0011-040013). We gratefully acknowledge Professor Sunghwan Kim in Kyungpook National University for allowing us to use the Q-Exactive instrument in his laboratory, and Yunju Cho for her assistance with the orbitrap experiments.

\section{Notes and references}

1 K. C. Aune and C. Tanford, Biochemistry, 1969, 8, 4586-4590. 2 K. Sasahara, M. Demura and K. Nitta, Proteins: Struct., Funct., Genet., 2002, 49, 472-482.

3 D. J. Segel, A. Bachmann, J. Hofrichter, K. O. Hodgson, S. Doniach and T. Kiefhaber, J. Mol. Biol., 1999, 288, 489-499.

4 L. L. Chen, K. O. Hodgson and S. Doniach, J. Mol. Biol., 1996, 261, 658-671.

5 P. Haezebrouck, M. Joniau, H. Vandael, S. D. Hooke, N. D. Woodruff and C. M. Dobson, J. Mol. Biol., 1995, 246, 382-387.

6 M. Hirai, S. Arai and H. Iwase, J. Phys. Chem. B, 1999, 103, 549-556.

7 P. Jennings and P. Wright, Science, 1993, 262, 892-896.

8 A. Litwińczuk, S. R. Ryu, L. A. Nafie, J. W. Lee, H. I. Kim, Y. M. Jung and B. Czarnik-Matusewicz, Biochim. Biophys. Acta, Proteins Proteomics, 2014, 1844, 593-606.

9 Y. Goto, L. J. Calciano and A. L. Fink, Proc. Natl. Acad. Sci. U. S. A., 1990, 87, 573-577.

10 M. M. Harding, D. H. Williams and D. N. Woolfson, Biochemistry, 1991, 30, 3120-3128.
11 M. Ohgushi and A. Wada, FEBS Lett., 1983, 164, 21-24.

12 H. S. Bose, R. M. Whittal, M. A. Baldwin and W. L. Miller, Proc. Natl. Acad. Sci. U. S. A., 1999, 96, 7250-7255.

13 B. Schuler and W. A. Eaton, Curr. Opin. Struct. Biol., 2008, 18, 16-26.

14 L. Fu, J. Liu and E. C. Y. Yan, J. Am. Chem. Soc., 2011, 133, 8094-8097.

15 G. Liu, A. Prabhakar, D. Aucoin, M. Simon, S. Sparks, K. J. Robbins, A. Sheen, S. A. Petty and N. D. Lazo, J. Am. Chem. Soc., 2010, 132, 18223-18232.

16 J. Fenn, M. Mann, C. Meng, S. Wong and C. Whitehouse, Science, 1989, 246, 64-71.

17 V. Katta and B. T. Chait, J. Am. Chem. Soc., 1991, 113, 85348535.

18 A. J. R. Heck, Nat. Methods, 2008, 5, 927-933.

19 R. H. H. v. d. Heuvel and A. J. R. Heck, Curr. Opin. Chem. Biol., 2004, 8, 519-526.

20 T. Wyttenbach and M. T. Bowers, J. Phys. Chem. B, 2011, 115, 12266-12275.

21 H. Shi, N. A. Pierson, S. J. Valentine and D. E. Clemmer, J. Phys. Chem. B, 2012, 116, 3344-3352.

22 J. W. Lee, S. W. Heo, S. J. C. Lee, J. Y. Ko, H. Kim and H. I. Kim, J. Am. Soc. Mass Spectrom., 2013, 24, 21-29.

23 S. J. C. Lee, J. W. Lee, T. S. Choi, K. S. Jin, S. Lee, C. Ban and H. I. Kim, Anal. Chem., 2014, 86, 1909-1916.

24 A. Dobo and I. A. Kaltashov, Anal. Chem., 2001, 73, 47634773.

25 A. K. Frimpong, R. R. Abzalimov, S. J. Eyles and I. A. Kaltashov, Anal. Chem., 2007, 79, 4154-4161.

26 Z. Hall and C. V. Robinson, J. Am. Soc. Mass Spectrom., 2012, 23, 1161-1168.

27 M. Šamalikova, I. Matecko, N. Muller and R. Grandori, Anal. Bioanal. Chem., 2004, 378, 1112-1123.

28 U. A. Mirza, S. L. Cohen and B. T. Chait, Anal. Chem., 1993, 65, 1-6.

29 L. Testa, S. Brocca and R. Grandori, Anal. Chem., 2011, 83, 6459-6463.

30 S. W. Heo, T. S. Choi, K. M. Park, Y. H. Ko, S. B. Kim, K. Kim and H. I. Kim, Anal. Chem., 2011, 83, 7916-7923.

31 J. Fernandez de la Mora, Anal. Chim. Acta, 2000, 406, 93-104.

32 L. Konermann, E. Ahadi, A. D. Rodriguez and S. Vahidi, Anal. Chem., 2013, 85, 2-9.

33 R. L. Grimm and J. L. Beauchamp, J. Phys. Chem. A, 2010, 114, 1411-1419.

34 U. A. Mirza and B. T. Chait, Int. J. Mass Spectrom. Ion Processes, 1997, 162, 173-181.

35 H. J. Sterling, C. A. Cassou, A. C. Susa and E. R. Williams, Anal. Chem., 2012, 84, 3795-3801.

36 H. Lin, E. N. Kitova, M. A. Johnson, L. Eugenio, K. K. S. Ng and J. S. Klassen, J. Am. Soc. Mass Spectrom., 2012, 23, 2122-2131.

37 S. Vahidi, B. B. Stocks and L. Konermann, Anal. Chem., 2013, 85, 10471-10478.

38 K. Pagel, E. Natan, Z. Hall, A. R. Fersht and C. V. Robinson, Angew. Chem., Int. Ed., 2013, 52, 361-365.

39 J. B. Hedges, S. Vahidi, X. Yue and L. Konermann, Anal. Chem., 2013, 85, 6469-6476. 
40 K. Breuker and F. W. McLafferty, Proc. Natl. Acad. Sci. U. S. A., 2008, 105, 18145-18152.

41 O. Skinner, F. McLafferty and K. Breuker, J. Am. Soc. Mass Spectrom., 2012, 23, 1011-1014.

42 T. Ly and R. R. Julian, J. Am. Chem. Soc., 2010, 132, 86028609.

43 D. M. Mao, K. R. Babu, Y. L. Chen and D. J. Douglas, Anal. Chem., 2003, 75, 1325-1330.

44 H. Sterling, M. Daly, G. Feld, K. Thoren, A. Kintzer, B. Krantz and E. Williams, J. Am. Soc. Mass Spectrom., 2010, 21, 17621774.

45 L. Konermann, A. D. Rodriguez and J. Liu, Anal. Chem., 2012, 84, 6798-6804.

46 B. A. Malcolm, S. Rosenberg, M. J. Corey, J. S. Allen, A. de Baetselier and J. F. Kirsch, Proc. Natl. Acad. Sci. U. S. A., 1989, 86, 133-137.

47 M. Hoshino, Y. Hagihara, D. Hamada, M. Kataoka and Y. Goto, FEBS Lett., 1997, 416, 72-76.

48 R. Diamond, J. Mol. Biol., 1974, 82, 371-391.

49 Y. O. Kamatari, T. Konno, M. Kataoka and K. Akasaka, Protein Sci., 1998, 7, 681-688.

50 S. J. Valentine, J. G. Anderson, A. D. Ellington and D. E. Clemmer, J. Phys. Chem. B, 1997, 101, 3891-3900.

51 D. S. Gross, P. D. Schnier, S. E. Rodriguez-Cruz, C. K. Fagerquist and E. R. Williams, Proc. Natl. Acad. Sci. U. S. A., 1996, 93, 3143-3148.

52 K. Kuwajima, Y. Hiraoka, M. Ikeguchi and S. Sugai, Biochemistry, 1985, 24, 874-881.

53 K. M. Downard, S. D. Maleknia and S. Akashi, Rapid Commun. Mass Spectrom., 2012, 26, 226-230.

54 B. J. McCullough, J. Kalapothakis, H. Eastwood, P. Kemper, D. MacMillan, K. Taylor, J. Dorin and P. E. Barran, Anal. Chem., 2008, 80, 6336-6344.

55 P. V. Konarev, V. V. Volkov, A. V. Sokolova, M. H. J. Koch and D. I. Svergun, J. Appl. Crystallogr., 2003, 36, 1277-1282.

56 D. Svergun, J. Appl. Crystallogr., 1992, 25, 495-503.

57 D. I. Svergun, M. V. Petoukhov and M. H. J. Koch, Biophys. J., 2001, 80, 2946-2953.

58 D. Svergun, C. Barberato and M. H. J. Koch, J. Appl. Crystallogr., 1995, 28, 768-773.

59 C. D. Putnam, M. Hammel, G. L. Hura and J. A. Tainer, $Q$. Rev. Biophys., 2007, 40, 191-285.

60 B. T. Ruotolo, J. L. P. Benesch, A. M. Sandercock, S.-J. Hyung and C. V. Robinson, Nat. Protoc., 2008, 3, 1139-1152.
61 M. F. Bush, Z. Hall, K. Giles, J. Hoyes, C. V. Robinson and B. T. Ruotolo, Anal. Chem., 2010, 82, 9557-9565.

62 B. Hess, C. Kutzner, D. van der Spoel and E. Lindahl, J. Chem. Theory Comput., 2008, 4, 435-447.

63 G. A. Kaminski, R. A. Friesner, J. Tirado-Rives and W. L. Jorgensen, J. Phys. Chem. B, 2001, 105, 6474-6487.

64 A. A. Shvartsburg and M. F. Jarrold, Chem. Phys. Lett., 1996, 261, 86-91.

65 M. F. Mesleh, J. M. Hunter, A. A. Shvartsburg, G. C. Schatz and M. F. Jarrold, J. Phys. Chem., 1996, 100, 16082-16086.

66 M. I. Catalina, R. H. H. van den Heuvel, E. van Duijn and A. J. R. Heck, Chem.-Eur. J., 2005, 11, 960-968.

67 R. Grandori, J. Mass Spectrom., 2003, 38, 11-15.

68 U. A. Mirza and B. T. Chait, Anal. Chem., 1994, 66, 2898-2904. 69 H. Vis, U. Heinemann, C. M. Dobson and C. V. Robinson, J. Am. Chem. Soc., 1998, 120, 6427-6428.

70 S. Shimoyama, A. Nagadoi, H. Tachiwana, M. Yamada, M. Sato, H. Kurumizaka, Y. Nishimura and S. Akashi, J. Mass Spectrom., 2010, 45, 900-908.

71 S. Merenbloom, T. Flick and E. Williams, J. Am. Soc. Mass Spectrom., 2012, 23, 553-562.

72 S. Merenbloom, T. Flick, M. Daly and E. Williams, J. Am. Soc. Mass Spectrom., 2011, 22, 1978-1990.

73 Y. Goto, N. Takahashi and A. L. Fink, Biochemistry, 1990, 29, 3480-3488.

74 S. Warnke, G. von Helden and K. Pagel, J. Am. Chem. Soc., 2013, 135, 1177-1180.

75 D. E. Kuehner, J. Engmann, F. Fergg, M. Wernick, H. W. Blanch and J. M. Prausnitz, J. Phys. Chem. B, 1999, 103, 1368-1374.

76 F. E. Kuhlmann, A. Apffel, S. M. Fischer, G. Goldberg and P. C. Goodley, J. Am. Soc. Mass Spectrom., 1995, 6, 1221-1225.

77 W. Z. Shou and W. Naidong, J. Chromatogr. B: Anal. Technol. Biomed. Life Sci., 2005, 825, 186-192.

78 S. A. Gustavsson, J. Samskog, K. E. Markides and B. Långström, J. Chromatogr. A, 2001, 937, 41-47.

79 N. B. Cech and C. G. Enke, Mass Spectrom. Rev., 2001, 20, 362-387.

80 H. P. Gregor, J. Belle and R. A. Marcus, J. Am. Chem. Soc., 1955, 77, 2713-2719.

81 A. Wexler, J. Res. Natl. Bur. Stand., Sect. A, 1976, 80, 775-785. 82 A. S. Coolidge, J. Am. Chem. Soc., 1930, 52, 1874-1887.

83 A. E. Potter and H. L. Ritter, J. Phys. Chem., 1954, 58, 10401042. 